\title{
A Novel Muon Detector for Borehole Density Tomography
}

Alain Bonneville, Richard T. Kouzes ${ }^{1}$, Jared Yamaoka

Pacific Northwest National Laboratory, Richland WA 99352 USA

Charlotte Rowe, Elena Guardincerri, J. Matthew Durham, Christopher L. Morris, Daniel C. Poulson,

Kenie Plaud-Ramos, Deborah J. Morley, Jeffrey D. Bacon

Los Alamos National Laboratory, Los Alamos, NM 87545 USA

James Bynes, Julien Cercillieux, Chris Ketter, Khanh Le, Isar Mostafanezhad, Gary Varner

University of Hawaii at Manoa, Honolulu, HI 96822 USA

Joshua Flygare, Azaree T. Lintereur

University of Utah, Salt Lake City, UT 84112 USA

\begin{abstract}
Muons can be used to image the density of materials through which they pass, including geological structures. Subsurface applications of the technology include tracking fluid migration during injection or production, with increasing concern regarding such timely issues as induced seismicity or chemical leakage into aquifers. Current density monitoring options include gravimetric data collection and active or passive seismic surveys. One alternative, or complement, to these methods is the development of a muon detector that is sufficiently compact and robust for deployment in a borehole. Such a muon detector can enable imaging of density structure to monitor small changes in density - a proxy for fluid migration - at depths up to $1500 \mathrm{~m}$. Such a detector has been developed, and Monte Carlo modeling methods applied to simulate the anticipated detector response. Testing and measurements using a prototype detector in the laboratory and shallow underground laboratory demonstrated robust response. A satisfactory comparison with a large drift tube-based muon detector is also presented.
\end{abstract}

Keywords: muon detection, subsurface fluid injection, carbon storage, GEANT4, plastic scintillator, density tomography

PACS: 29.40.-n, 29.40.Mc, 93.85.-q, 89.30.A-

\footnotetext{
${ }^{1}$ Corresponding Author: Phone: +1-509-372-4858; E-mail: rkouzes@pnnl.gov
} 


\section{Introduction}

The determination of the density distribution of material in the Earth's subsurface, and the evolution of density as a function of time, has the potential to provide a sensitive, cost-effective, and precise monitoring technique to determine field-scale displacement of reservoir fluid induced by injection or production of liquids or gases. Geological carbon storage, natural gas storage, enhanced oil recovery, compressed air storage, aquifer storage and recovery, waste water storage and oil and gas production are examples of application areas. It is thus crucial to monitor in quasi-real time the behavior of these fluids, and several monitoring techniques can be used. Among them, those that track density changes in the subsurface are the most relevant. To date, the only way to collect direct and quantitative density distribution information is to measure changes in the Earth's gravitational field, known as time-lapse gravity measurements. Time-lapse gravity has been used for more than 50 years. Substantial developments over the last decade in gravimeter technology, as well as the advent of a precise Global Positioning System (GPS), have led to improvements in differential microgravity measurements; however, this technique is limited in that it only provides discrete values of the gravity field anomaly that represent the integral of the density distribution, and it is by nature an underdetermined problem.

Cosmic ray muon tomography (or muography) can provide a complete and precise image of the density distribution in the subsurface due to the dependence of the loss of flux on the varying density through which the muon passes. This approach has the potential to become a direct, real-time, and low-cost method for monitoring fluid displacement in subsurface reservoirs. Taking the example of geological carbon storage, such a method will allow monitoring the $\mathrm{CO}_{2}$ concentration by watching for density changes over time as the $\mathrm{CO}_{2}$ is injected and replaces brine. The muon detector for such an application needs to have sufficient angular resolution as well as high efficiency due to the low muon flux values at the depths at which $\mathrm{CO}_{2}$ will be stored. The time scale for collecting a statistically significant number of muon events depends exponentially on depth, and linearly on the detector area. Signal to noise depends on the square root of the collection time. This means that a monitoring system for carbon sequestration requires a chain of many detectors (perhaps approximately 20) in a horizontal borehole beneath the site, and limits the use to depths less than approximately $1300 \mathrm{~m}$. Data collection times will be days to weeks, but this is not a limitation since the changes in geology are on a longer time scale.

Within the New Subsurface Signals pillar of the U.S. DOE crosscut program SubTER, Pacific Northwest National Laboratory (PNNL), in collaboration with Lawrence Livermore National Laboratory, Los Alamos National Laboratory (LANL), Sandia National Laboratory, University of Utah, University of Hawaii and Paulsson Inc., has developed a borehole muon detector (BMD) to evaluate the densitydependent attenuation of the cosmic ray muon flux at depth. The BMD development has substantially reduced the size of muon detectors [1][2], providing confidence that borehole-deployable systems are technically and economically feasible. Following successful tests in surface laboratories, the BMD was deployed in the PNNL Shallow Underground Laboratory and in a tunnel at LANL where the data collected were compared to data collected by a large LANL muon detector instrument. After a brief introduction to the principles of muon tomography, the design of the BMD prototype, as well as the first results of the initial series of tests and benchmarking, is presented.

\section{Muon Tomography}

Muons are generated in the upper atmosphere from cosmic ray interactions, and penetrate into the Earth at multiple angles, being attenuated by the different stratigraphic units depending on their densities. By measuring the muon flux at different depths in vertical, inclined, or horizontal wells, the attenuation of the muon signal due to the different stratigraphic units, or the fluids contained within these units, can be determined. These measurements can be performed continuously to identify and interpret variations in density and fluid content as a function of time, and be processed and interpreted jointly with other 
geophysical data (passive and active seismic because seismic waves are also sensitive to density, gravity, etc.), thereby improving spatial resolution and reducing uncertainty.

Muon radiography has been used to successfully image the displacement of magma in active volcanoes [3][4][5] with unprecedented detail using large detectors deployed at the surface. It was shown that the Tanaka detector, with a resolution of $10 \mathrm{mrad}$, was capable of imaging a volcano $1 \mathrm{~km}$ away to within approximately $10 \mathrm{~m}$. Muon density imaging also has demonstrated potential for non-proliferation efforts to detect hidden nuclear weapons or nuclear material in vehicles [6]. Using scattering angles, significant quantities of very high-density material were detected inside cargo within a few minutes. Muon density imaging can also be used as a means to non-invasively monitor dry storage casks for spent nuclear fuel [7]. It was shown that, using either a small detector from multiple angles or a large detector that surrounds the cask, the special nuclear material content of the cask could be validated with high accuracy within two days.

The goal of producing tomographic density maps of $\mathrm{CO}_{2}$ reservoirs presents new considerations surrounding the depth of deployment and associated reduction in muon flux. Although the flux rapidly decreases with depth [8][9], simulations indicate that the muon technique will have sufficient sensitivity to effectively map density variations caused by fluid displacement at great depths. For example, the

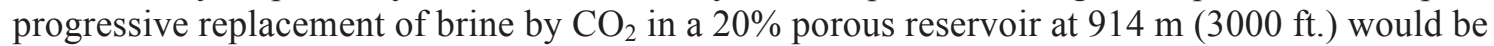
detectable over periods of weeks to months.

The primary technical challenge preventing deployment of muon detection technology in the subsurface is the lack of miniaturized muon-tracking detectors capable of fitting in standard boreholes that will resist the harsh underground conditions for long periods of time. The development of a miniaturized muontracking detector deployed in a borehole is guided by strict requirements of size, ability to withstand an underground environment, data transmission rate limitations, background effects, as well as performance requirements. A typical borehole has a deployment diameter no larger than $17.8 \mathrm{~cm}$ (7'); hence, the detector, with all associated equipment, needs to be placed completely inside a stainless steel housing that does not exceed $17.8 \mathrm{~cm}$ (or less) in diameter.

The data transmission will occur through a cable connecting the detector to the surface. The cable will transmit power to the detector and transfer data from the detector to the surface. In the future when multiple detectors are needed in a tether, this approach can sequence detectors together and recover all the data. The assembly must have the ability to withstand the harsh environments present in the borehole, including pressure, heat, water and corrosive chemicals.

Discrimination of background radiation impacts on the detector from gamma rays and beta particles will be important to reach the required statistical quality of the data. Using GEANT4 simulations [10], it was shown that the background gamma rays from typical natural sources present at depth would be completely mitigated by placing an energy threshold of approximately $150 \mathrm{keV}$ on each muon event [11]. Beta and alpha particles were shown to be unable to penetrate the stainless steel housing or generate any coincidence events in the detector.

\section{Prototype Borehole Muon Detector}

The design for the BMD to be used in deep boreholes to monitor muon flux and the angle of incidence, is based on scintillating rods with fiber readout, pixelated silicon photomultiplier (SiPM), and integrated threshold and coincidence electronics. The BMD design is intended for a horizontal borehole positioned below the geology to be monitored, with integrated electronics and data transmission to the surface for long term monitoring applications. A prototype BMD [11] was built with four layers of $1 \mathrm{~cm}$ square polystyrene scintillator rods coated with $\mathrm{TiO}_{2}$ reflector (provided by Fermi National Laboratory), as shown in Figure 1. The BMD has dimensions of $15 \mathrm{~cm}$ wide, $68 \mathrm{~cm}$ long, and $8 \mathrm{~cm}$ high, designed to fit into a $17.8 \mathrm{~cm}$ (7-in.) borehole. Each rod includes a 2-mm diameter wavelength shifting fiber from Saint Gobain (Hiram, OH) glued in the center of the rod, which leads to a SiPM to pick up the scintillation light 
generated as a result of a muon transit. Top to bottom, the layers are alternating rows in the $\mathrm{x}$ and $\mathrm{y}$ directions, providing an internal coordinate system (the layers of long rods are seen in Figure 1). There are long rods $(68 \mathrm{~cm}$ each) and short rods $(15 \mathrm{~cm}$ each) in the BMD with the top pair and bottom pair of layers separated by $5.8 \mathrm{~cm}$ center-to-center. While the two long-rod layers were fully populated with 15 rods each, the two short-rod layers were only partially populated with 30 rods each (centered on the long rods), for a total of 90 rods. Background was rejected by requiring a four-fold coincidence between the layers (within a $140 \mathrm{~ns}$ coincidence window to produce a hardware trigger). The angle of the muon transit in the $\mathrm{x}$ and $\mathrm{y}$ directions can be computed by extrapolating between discrete rod hits by weighting the energy deposition in multiple rods. The angular resolution obtained with the BMD was approximately 3 degrees, which is adequate for the intended application. The intrinsic efficiency of the detector is close to $100 \%$, as validated with comparison to the known muon flux.

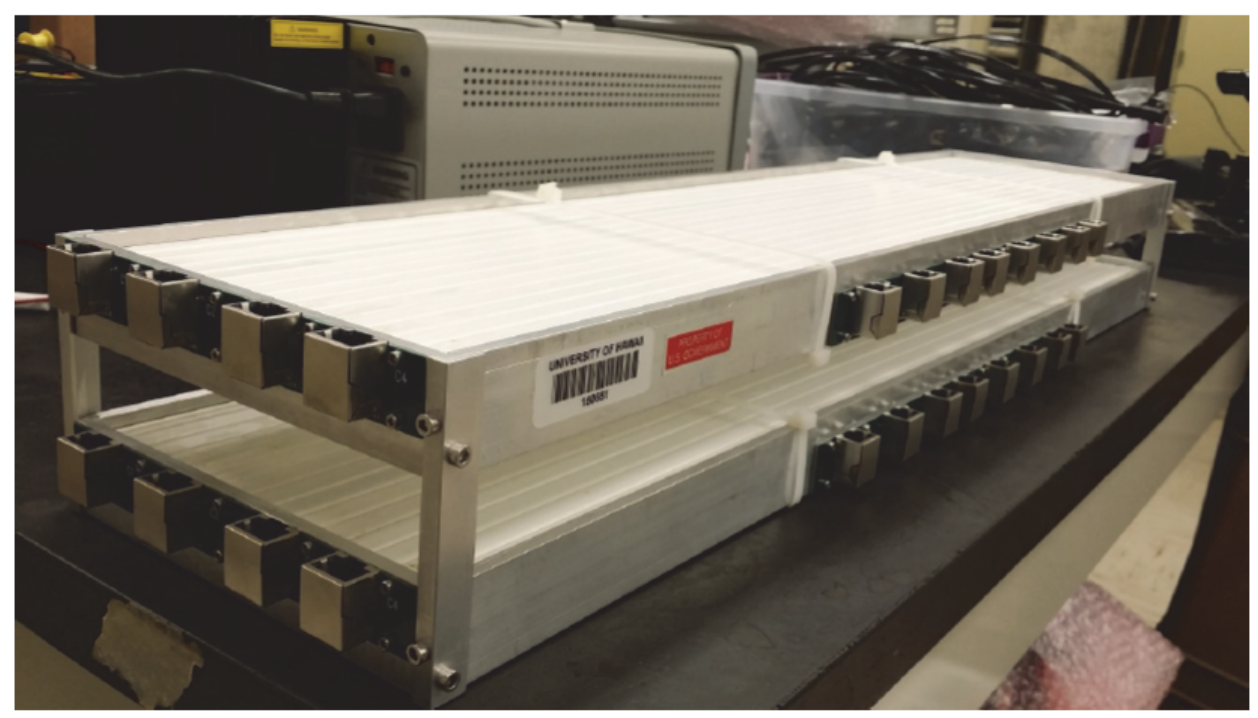

Figure 1. Prototype BMD assembly showing layers of polystyrene rods and SiPM photodetector assemblies.

\section{Electronics, Data Acquisition and Format}

The University of Hawaii designed and implemented the readout electronics for the BMD. Custom made hardware and firmware were used to collect the data and provide preliminary processing such as lowenergy thresholds. Figure 2 shows the readout electronics box that contains the low and high voltage power supply, motherboard (chassis), interconnect board and digitizer daughter card. The BMD output enters the interconnect board. 


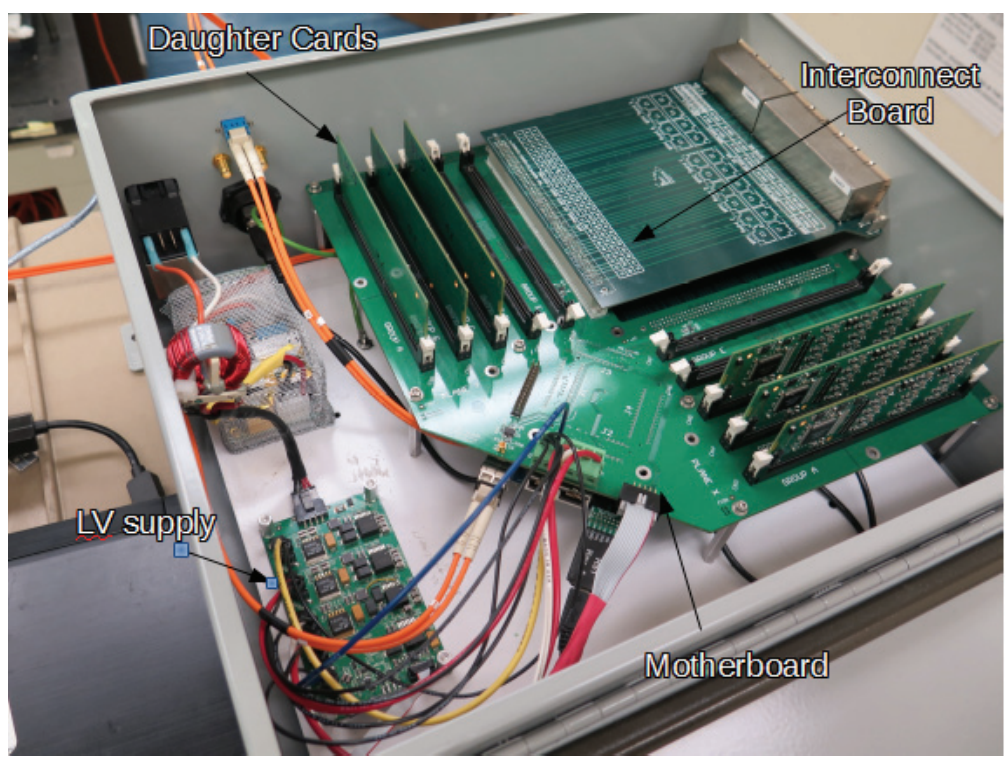

Figure 2. Contents of the readout electronics box. Labeled in the picture is the interconnect board, the motherboard (chassis), the LV power supply, and the digitizer daughter cards.

The power supply has a standard 100-220 VAC input and generates the four required voltages to bias the SiPM (Hamamatsu $3 \mathrm{~mm}$ by $3 \mathrm{~mm}$ model S12572-050P) multi-pixel photon counters (MPPCs). The readout system communicates with a personal computer (PC) using a standard fiber optic Ethernet link through a generic media converter. This keeps the readout design simple and eliminates dependency on custom data aggregation boards and devices. Figure 3 shows the entire data acquisition assembly consisting of: PC, electronics enclosure (Figure 2), JTAG USB, USB to RJ45 adapter, media converter, fiber optic cable, power cord, and the BMD (Figure 1) in a light-tight pelican case.

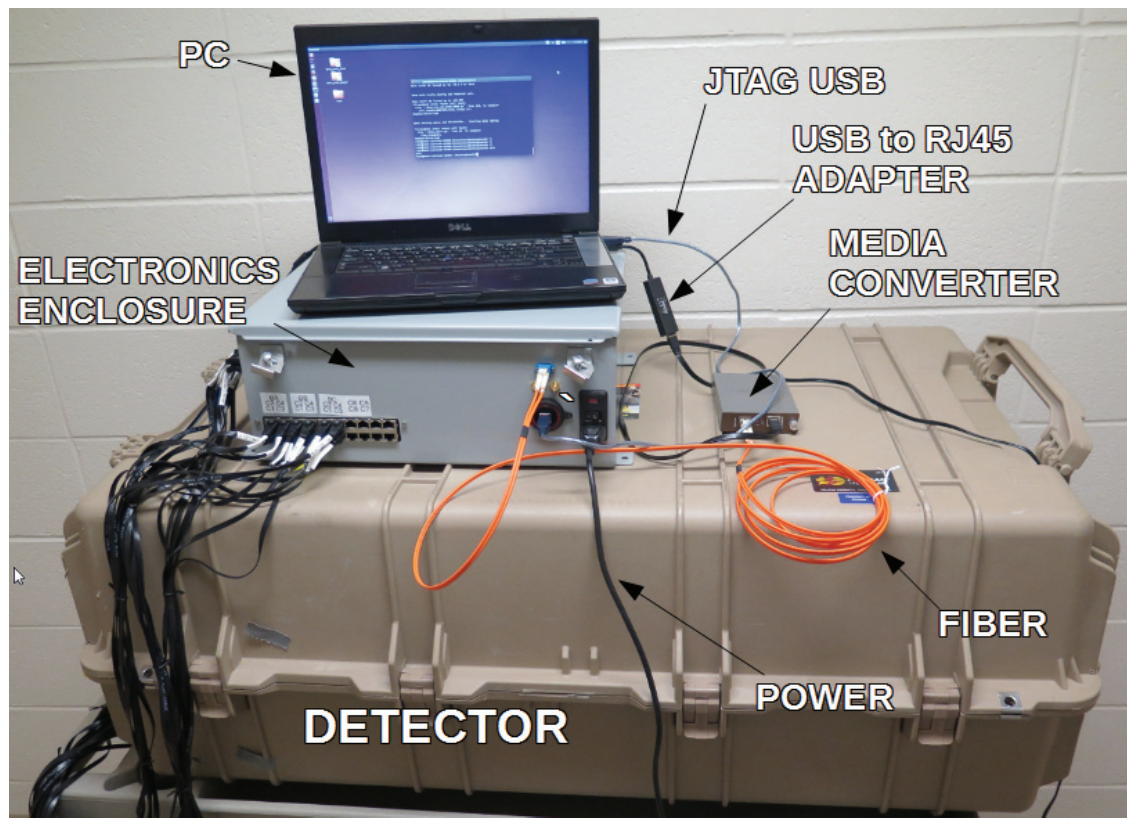

Figure 3. Complete data acquisition setup including the PC (top), electronics enclosure (middle), and the detector in a light-tight pelican case (bottom). 
A USB link exists to program the onboard field-programmable gate array (FPGA) using the Joint Test Action Group (JTAG) interface. The FPGA device is in the Xilinx Spartan 6 family and is installed on a separate circuit board (standard controls and readout device) beneath the motherboard shown in Figure 2. Each of the boards was designed at the Instrument Development Lab (IDLAB) in the Department of Physics and Astronomy at the University of Hawaii at Manoa. They were all fabricated by a contractmanufacturing house and then tested and integrated at the IDLAB.

The core of the electronics readout system is the TARGETX digitizer chip or Application Specific Integrated Circuit (ASIC). This chip was designed at IDLAB and manufactured with a standard $250 \mathrm{~nm}$ CMOS fabrication process. The TARGETX digitizer is capable of digitizing 16 channels of data at 1 $\mathrm{GSa} / \mathrm{s}$ each. It also includes circuitry for signal amplification, conditioning and programmable threshold discrimination used to form a global trigger. Companion digital to analog converter components provides individual channel gain tuning of the $\sim 70$ VDC signal for biasing the MPPCs. Each SiPM bias was set such that, at 5 photoelectrons (pe), the dark count rate was $1.5 \mathrm{KHz}$, while the single channel threshold was approximately 20 pe. The gain of the detector does have some temperature dependence, which will be controlled in the next version of the detector. A muons deposit approximately $2 \mathrm{MeV}$ in each scintillator rod. The first level hardware threshold was kept fairly low on the pulse height distribution to indicate the detection of a photon. Combined with the four-fold firmware threshold and coincidence requirement to reduce accidentals to a very low level, the detection efficiency for a muon that passed all four detection layers was very close to $100 \%$, with little variation in the efficiency over time or location.

Custom readout firmware (FW) and software was written for this application. The readout FW is code written in VHDL (VHSIC Hardware Description Language) to control the FPGA. The FPGAs control the digitizers and signal readout commands when an event is detected. After the event is read out, the FPGA creates a user datagram protocol (UDP) packet and sends it to the PC. The software receiving the signal from the FPGA on the PC is a combination of Python and $\mathrm{C}++$ code that waits for data packets as well as sends commands back to the FPGA. The packets, once received by the PC, are recorded to disk into a time-stamped data format for future processing.

The data from the BMD is formatted into a standard file format to be analyzed later by post-processing scripts. Each event has a header line that contains the run number, event number, number of hits in that event, and the time of the beginning of the event. Each event includes one line for each bar that is hit, and is described by the bar layer, axis, bar number (strip), Unix time of hit, and charge deposited. This information provides the post-processing software with enough detail to recreate a muon track through the four layers as a vector in space. The charge is used to average the hit location across multiple bars in a single layer, each position weighted by the charge received.

\section{Simulations}

A model of the BMD was made and simulated extensively [11] to predict detector response and performance as well as background radiation discrimination. The Monte Carlo code GEANT4 [10] was used for simulations. They were compared with experimental data for validation, and the results are shown in Figures 4 and 5, where the horizontal axes are in degrees and the vertical axis is normalized to one. Figure 4 shows a comparison of the angular distribution in both coordinate directions for a $\cos ^{2}$ distribution (outer mesh) and the simulated detector response above ground (solid surface). The detector response decreases with angle more rapidly than a $\cos ^{2}$ distribution due to the limited physical size of the detector active area $(15 \mathrm{~cm} \times 30 \mathrm{~cm})$. This simulated detector response distribution was verified with detector measurements, as shown in Figure 5 of a $\cos ^{2}$ distribution (outer mesh) compared to measurement above ground (solid surface). The BMD response had a mean at approximately zero with $\mathrm{x}$ and y standard deviations of 23.6 and 25.9 , respectively. The simulated response also had a mean at approximately zero with $\mathrm{x}$ and $\mathrm{y}$ standard deviations of 24.3 and 26.4 , respectively. 


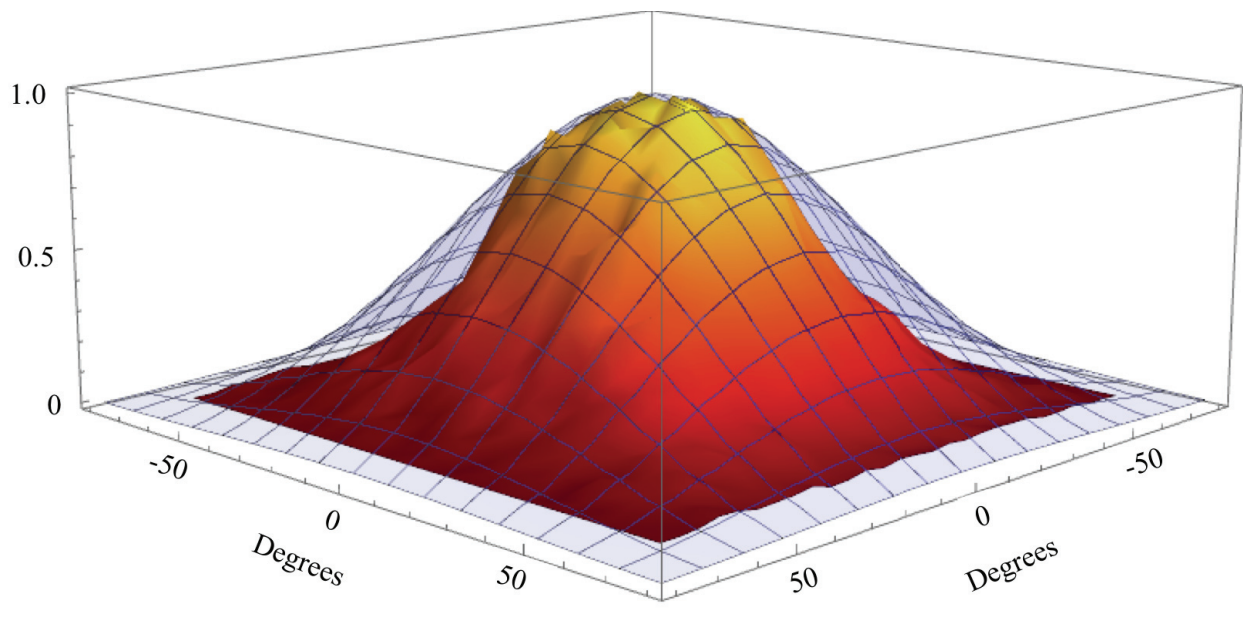

Figure 4. A GEANT4 simulation of the expected detector response compared to a $\cos ^{2}$ distribution.

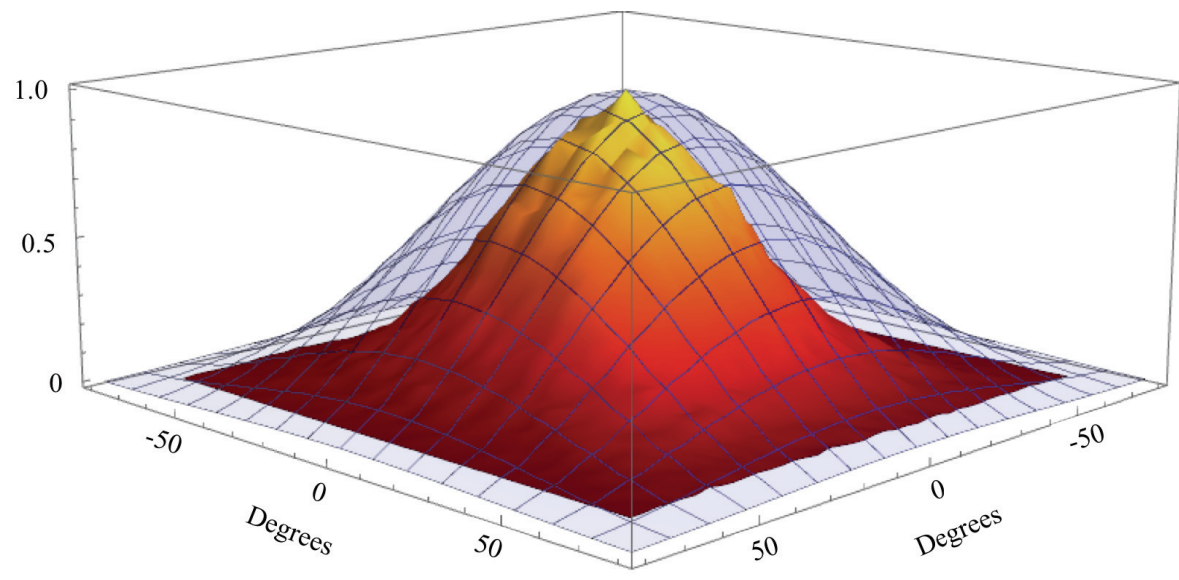

Figure 5. Measured BMD response compared to $\cos ^{2}$ distribution.

Additional simulations were performed in this work to determine the expected muon flux for the underground measurement locations. The simulation models were used to predict the muon flux at the PNNL Shallow Underground Laboratory and the TA41 tunnel at LANL. While two specific sites were used for this model, the procedure developed and described is adaptable to other sites. To ensure a realistic simulation, it was important to have a representative muon flux model. Muon angular and energy spectra change as the muons penetrate into the earth. The initial energy and angular spectra are simulated using the CRY Monte Carlo (MC) program [12]. CRY provides muon spectra and trajectories at the Earth's surface and includes latitude, altitude, and time of year as parameters. The CRY muon energy spectrum was validated against experimental measurements [12]. Figure 6 shows the angular distribution generated by CRY at the surface of the Earth at PNNL (36 deg. N. Latitude). 


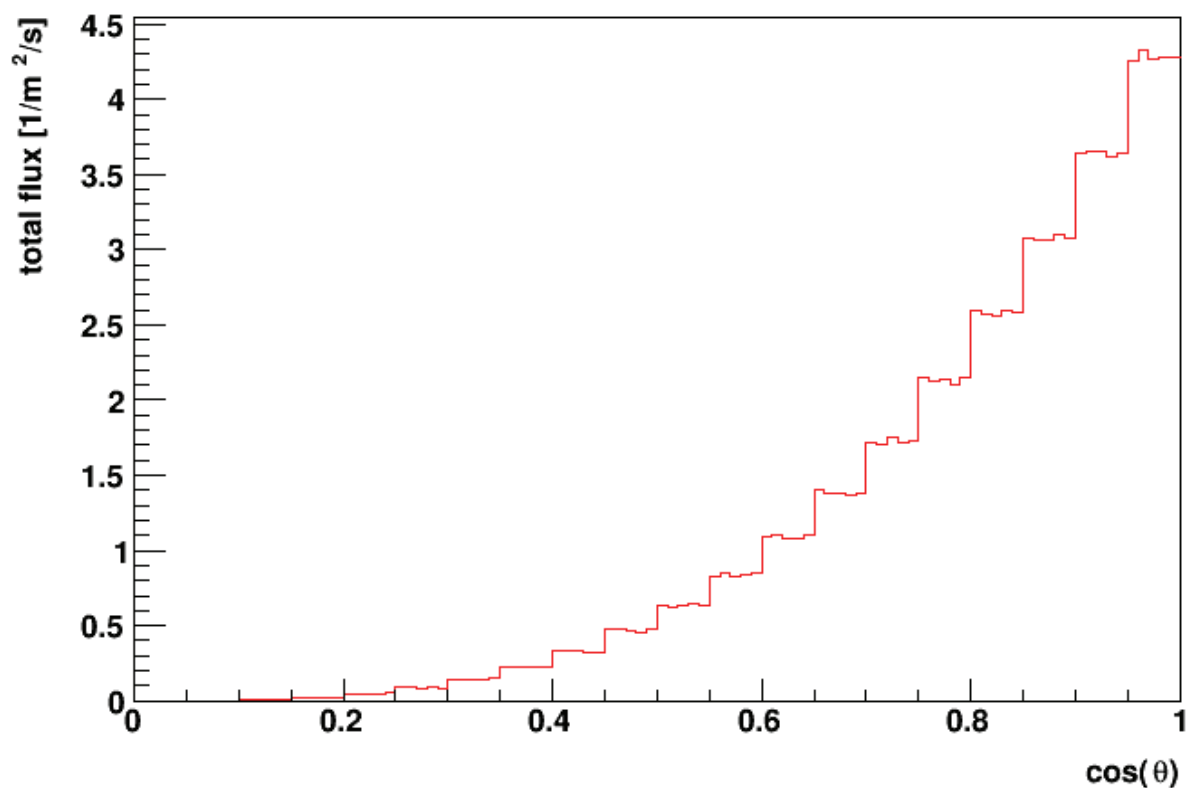

Figure 6. CRY Muon angular distribution at the surface of the Earth at PNNL (36 deg. N. Latitude).

A model of the PNNL Shallow Underground Laboratory was used and constructed in GEANT4. Figure 7 shows the model as a perspective-view. The overall size of the simulated environment is approximately $79 \mathrm{~m}$ by $40 \mathrm{~m}$ by $20 \mathrm{~m}$. The fill material is shown in brown and green, and the blue indicates the two vertical entry shafts and the main room. Blue areas are open and are simulated as air with a density of $1.29 \mathrm{mg} / \mathrm{cm}^{3}$. The fill and earth material are defined $\mathrm{as}^{\mathrm{CaCO}} 3$ with a density of $2.80 \mathrm{~g} / \mathrm{cm}^{3}$.

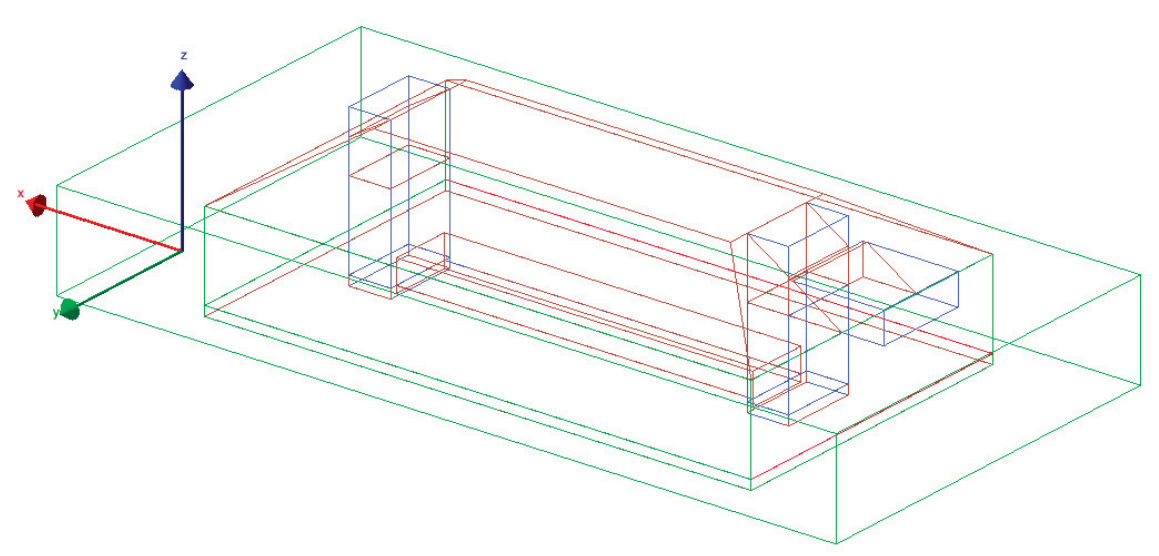

Figure 7. GEANT4 model of the PNNL Shallow Underground Laboratory showing a perspective-view.

Using the full cosmic muon spectrum as generated by CRY, a number of muon tracks corresponding to several days of exposure were generated. Figure 8 shows the total modeled flux at the bottom of the PNNL Shallow Underground Laboratory, $11 \mathrm{~m}$ beneath the surface (in the $\mathrm{z}$ axis). The shafts are clearly visible as flux hot spots and the lab space running between them also causes a noticeable increase in flux. 


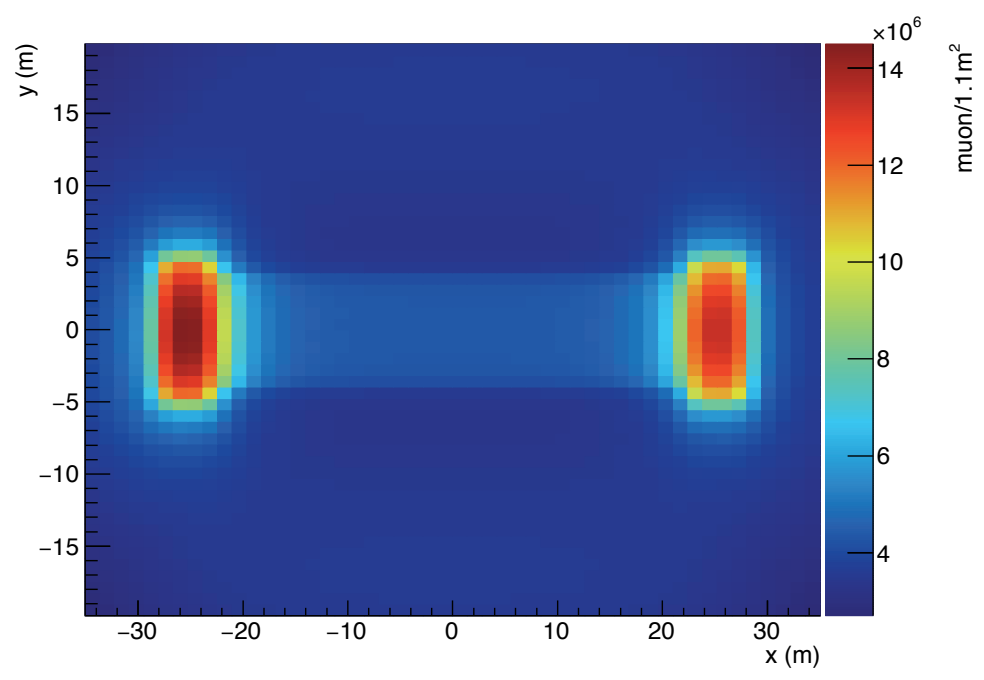

Figure 8. Total modeled flux at the bottom of the facility at $11 \mathrm{~m}$ below the surface. Exposure time is 48 hours.

A simplified detector model consisting of two $30 \mathrm{~cm}$ by $15 \mathrm{~cm}$ planes separated by $4 \mathrm{~cm}$ was used to approximate the detector acceptance. The BMD was placed just outside the lab space in the Shallow Underground Laboratory at approximately $\mathrm{x}=-20 \mathrm{~m}$, close to the shaft. Data collected at this location were used to produce several flux images and to explore imaging sensitivity. To produce the images shown in Figure 9, the simulated muon tracks that passed through all four layers of the simplified detector model were extended to the surface and mapped as a flux density. Figure 9 presents these projections at the surface $(\mathrm{z}=0 \mathrm{~m})$.
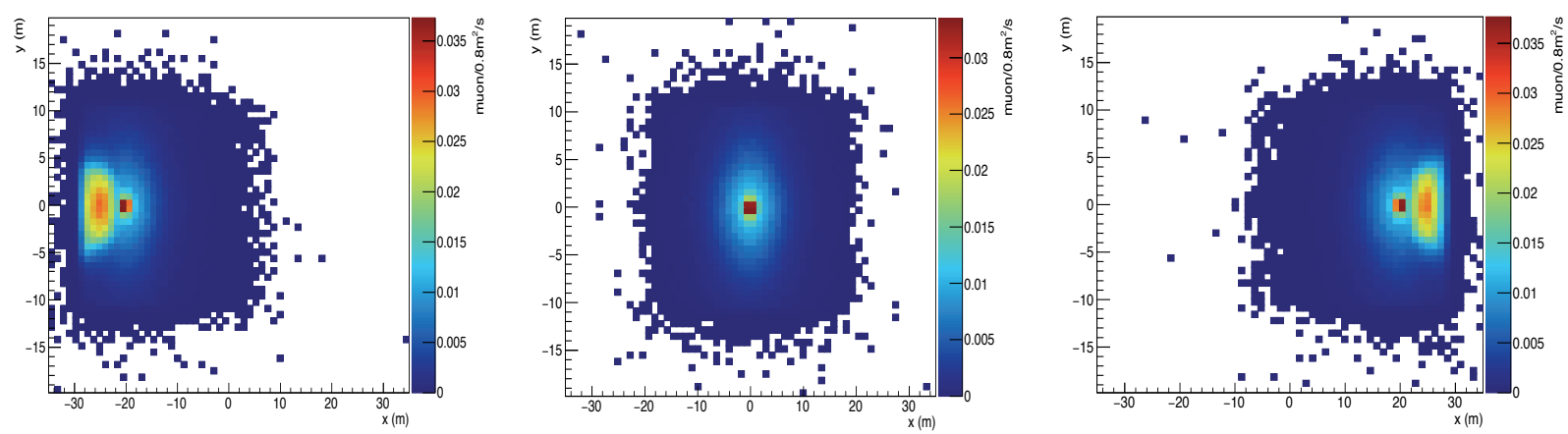

Figure 9. Projections to the surface at $\mathrm{z}=0 \mathrm{~m}$ of simulated muon tracks for the detector model placed in the main stairwell at $x=-20 \mathrm{~m}$ (left), the lab space at $\mathrm{x}=0 \mathrm{~m}$ (center), and the emergency stairwell at $\mathrm{x}=20 \mathrm{~m}$ (right).

Because of the simulations performed for this PNNL Shallow Underground Laboratory, it was predicted that only a few days of data taking with the prototype BMD would be required to image the shaft closest to the BMD location. Simulations were performed to determine the flux at various locations in the second test site, the TA41 Tunnel at LANL. The tunnel is a decommissioned drift driven into the Bandelier Tuff units in Los Alamos Canyon, with a floor approximately $90 \mathrm{~m}$ below the mesa top above, and extending approximately $90 \mathrm{~m}$ into the side of the mesa (see Figure 10). Figure 11 shows the site as it appeared in 1991 [13]. 


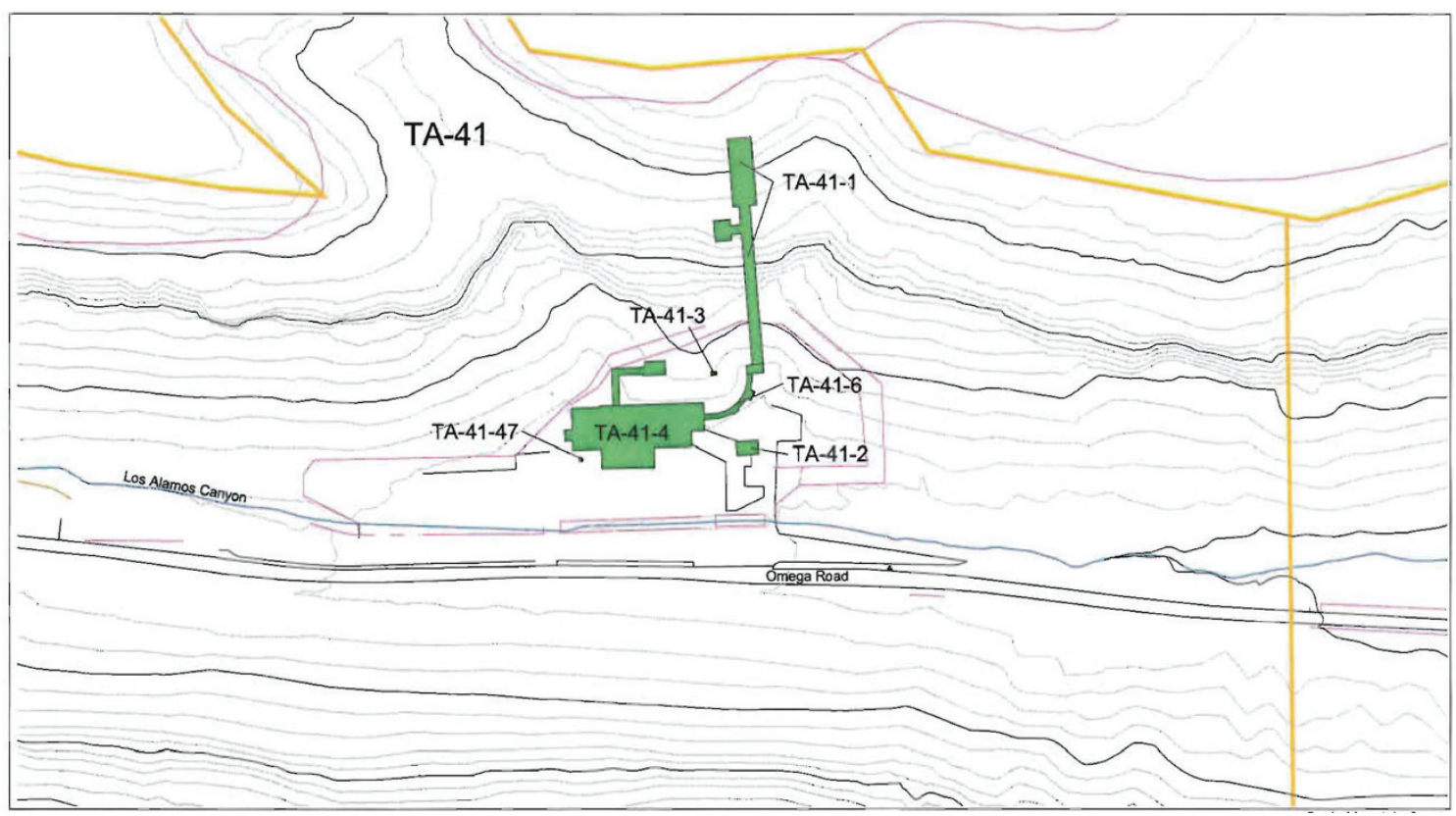

Figure 10. Layout of LANL Tunnel (TA-41-1) with topographic contours [13].

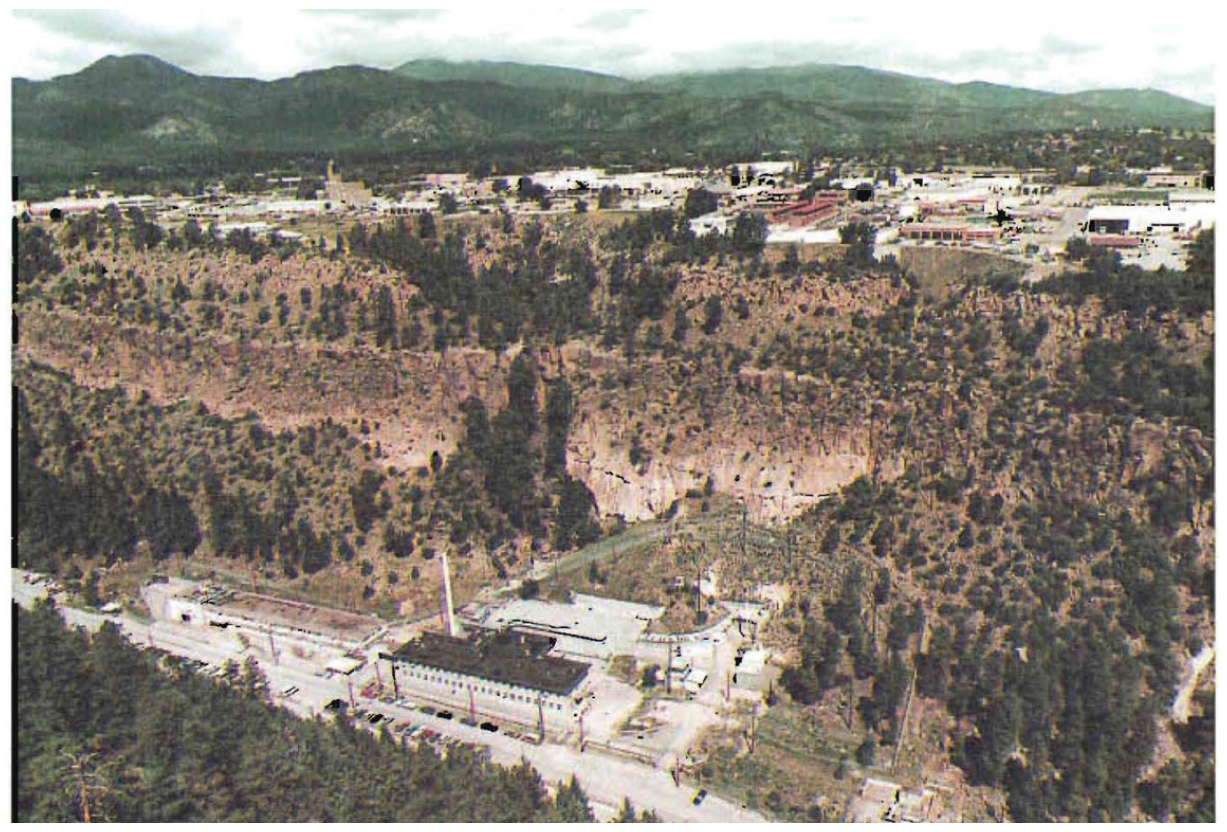

Figure 11. LANL Tunnel site as it appeared in 1991 [13].

A software framework was developed to take topographical data from a high precision LIDAR scan with a $30.5 \mathrm{~cm}$ (1 ft.) grid spacing, and lower the resolution to a $10 \mathrm{~m}$ grid to be loaded into the GEANT4 model. This method also allows for localized tuning of the density. For this simulation, the overburden was assumed to be $\mathrm{CaCO}_{3}$ rock with a density of $2.0 \mathrm{~g} / \mathrm{cm}^{3}$ (the actual rock is silicate volcanic tuft). Figure 12 shows the LIDAR scan and the associated GEANT4 model of the TA41 Tunnel. The shape of 
the mesa can be clearly seen from either rendering, showing the main valley and some of the finer structure of the cliff (the $\mathrm{x}$ coordinate is parallel to the mesa and the y coordinate is into the mesa).
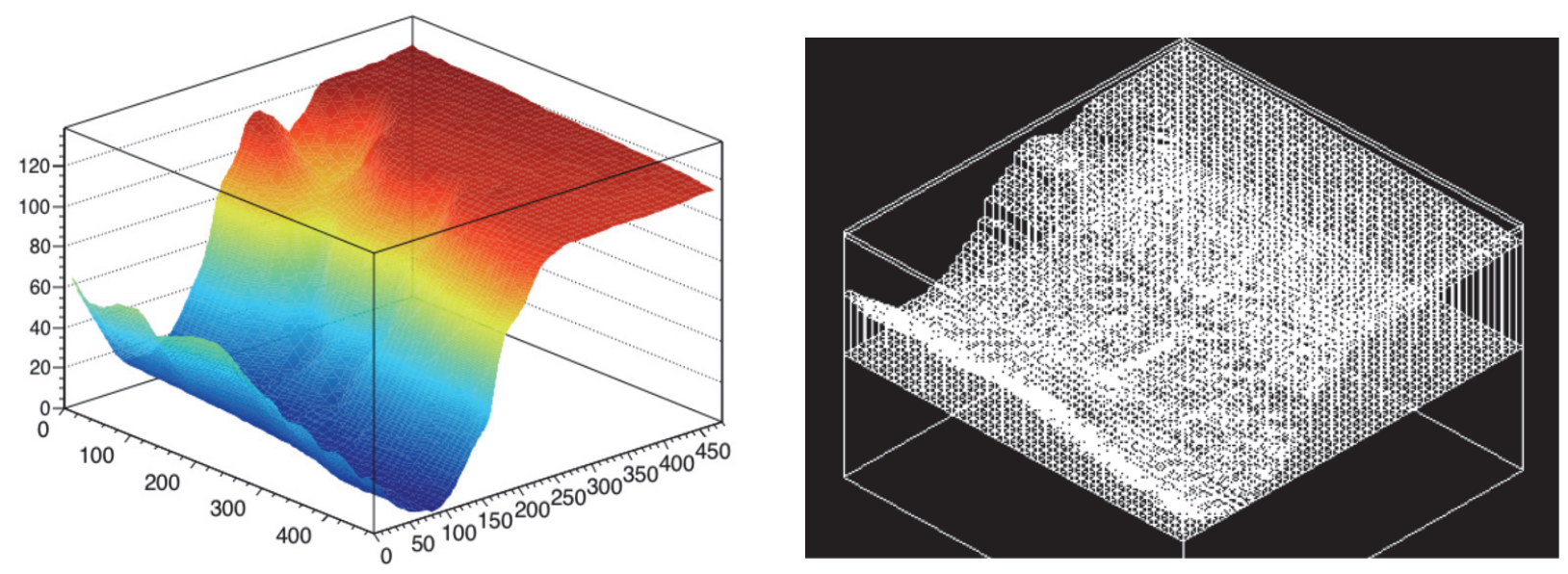

Figure 12. Topographical representation of the LANL TA41 test site in LIDAR (left) with $30.5 \mathrm{~cm}(1 \mathrm{ft}$.) grid spacing, and in GEANT4 (right) with $10 \mathrm{~m}$ grid spacing (units on all axes are in $\mathrm{m}$ ).

The GEANT4 simulation was used to produce a total flux plot at the bottom of the Tunnel site. Data from these simulations was used to compare the experimental flux with the predicted flux at various positions in the tunnel.

\section{Data collected in surface laboratory}

Data from the BMD were recorded on a laptop computer and then transferred to a server for post-analysis. The data were subject to three levels of thresholds. The first threshold is at the hardware level to determine whether a photodetector was activated. The second threshold is at the firmware level to accept the coincident bar hit. The third threshold is applied in the analysis software to further reduce the detector background.

Data analysis codes were written to read the data files and to generate histograms of the incident muon angle and flux. The program reads the data file event by event, requiring a four-fold coincidence between the four layers of the BMD, and compiles histograms of hit angles and the number of hits to each bar. The hit angles are computed by extrapolating the position in each layer based on charge weighting across bars (if multiple bars are hit) and then computing the $\mathrm{x}$ and $\mathrm{y}$ angles and the resulting zenith angle. If a single bar within a layer is hit, the bar position is randomized to a location within the bar width. This was shown to significantly increase the plotting resolution, as nearly $60 \%$ of all hits per layer are registered as single bar hits. Figure 13 shows example histograms of raw hits (with the firmware coincidence requirement) to each bar in the BMD, grouped by layer. The first group is the lower 15 long bars, followed by the lower 30 short bars, the upper 15 long bars, and the upper 30 short bars. These histograms illuminate variation in the efficiency of the bars that is removed by software coincidence and threshold adjustments during analysis. 


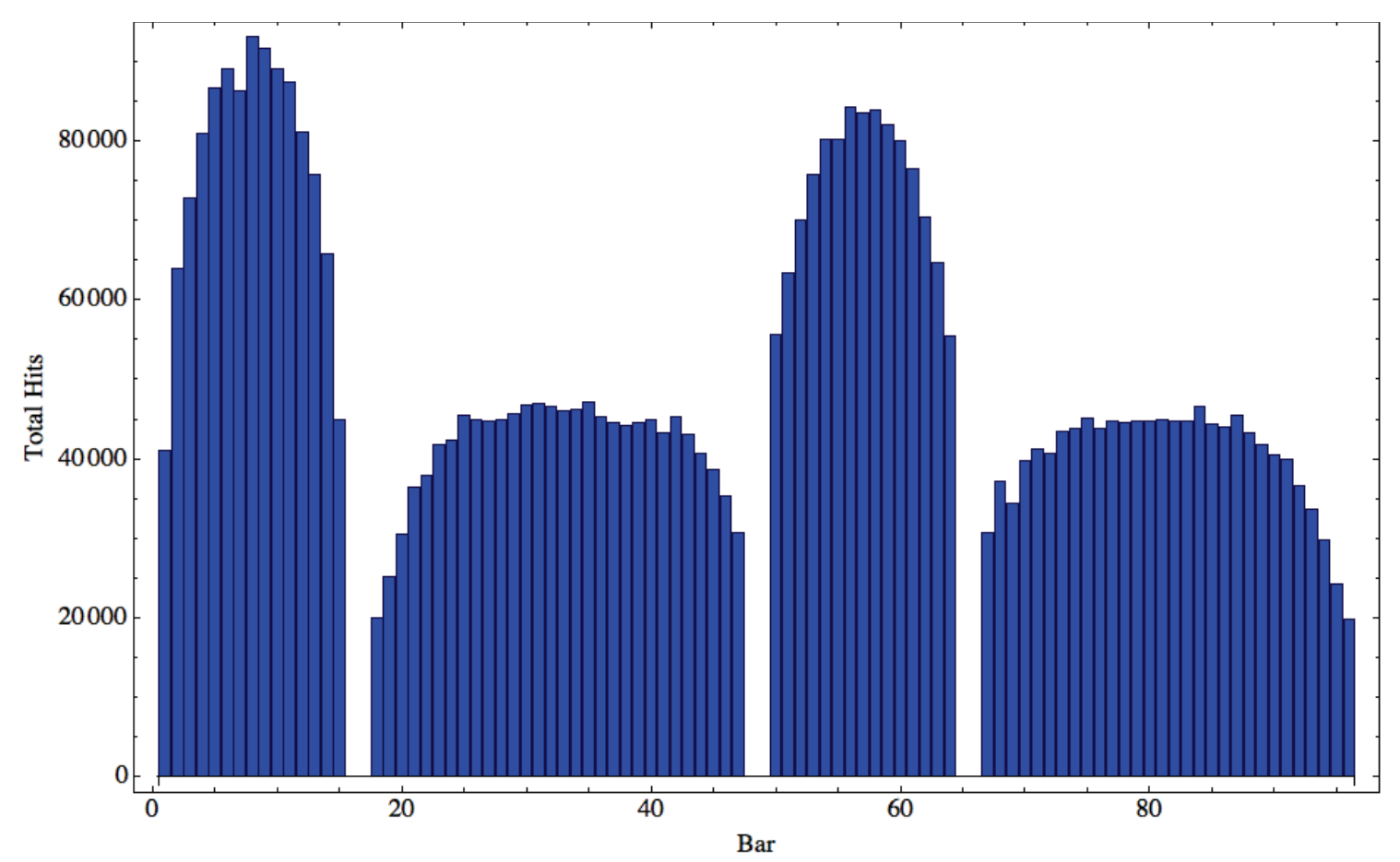

Figure 13. Histograms of bar hits for the four layers of scintillator bars.

The three-dimensional histogram of incident muon angle was smoothed to reduce variation caused by the discrete nature of the bar geometry. The amount of smoothing applied affects how much can be derived from the data, and is a balance of the degree of smoothing required to reduce the variations from the detector, while retaining any important details in the angular flux. Figure 14 is an example of a twodimensional histogram from data obtained with the prototype BMD, showing about $128 \mathrm{k}$ events.

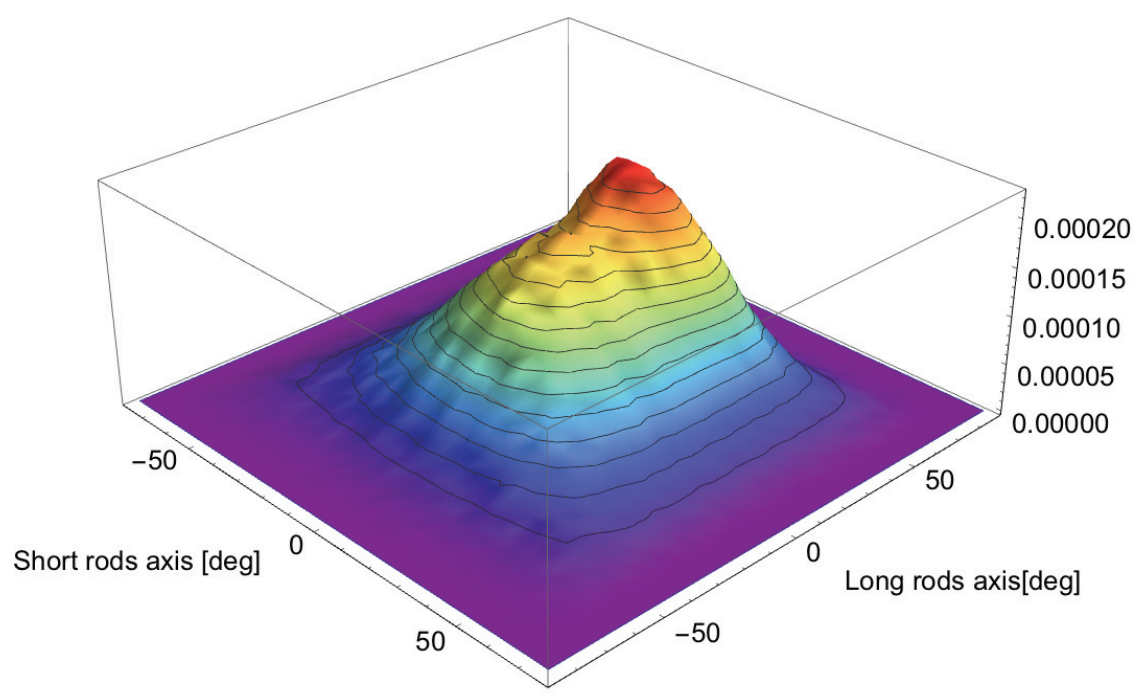

Figure 14. Two-dimensional histogram of data from the BMD, where the vertical axis is intensity in arbitrary units. Two degree smoothing of the data was used. 
Figure 15 projects the muon flux shown in Figure 14 onto a plane 30 meters above the BMD to obtain an image of spatial density variations. It is represented by a density histogram in which the color indicates the muon intensity in that projected spatial bin. This technique is useful in approximating a relative column density in the BMD overburden.

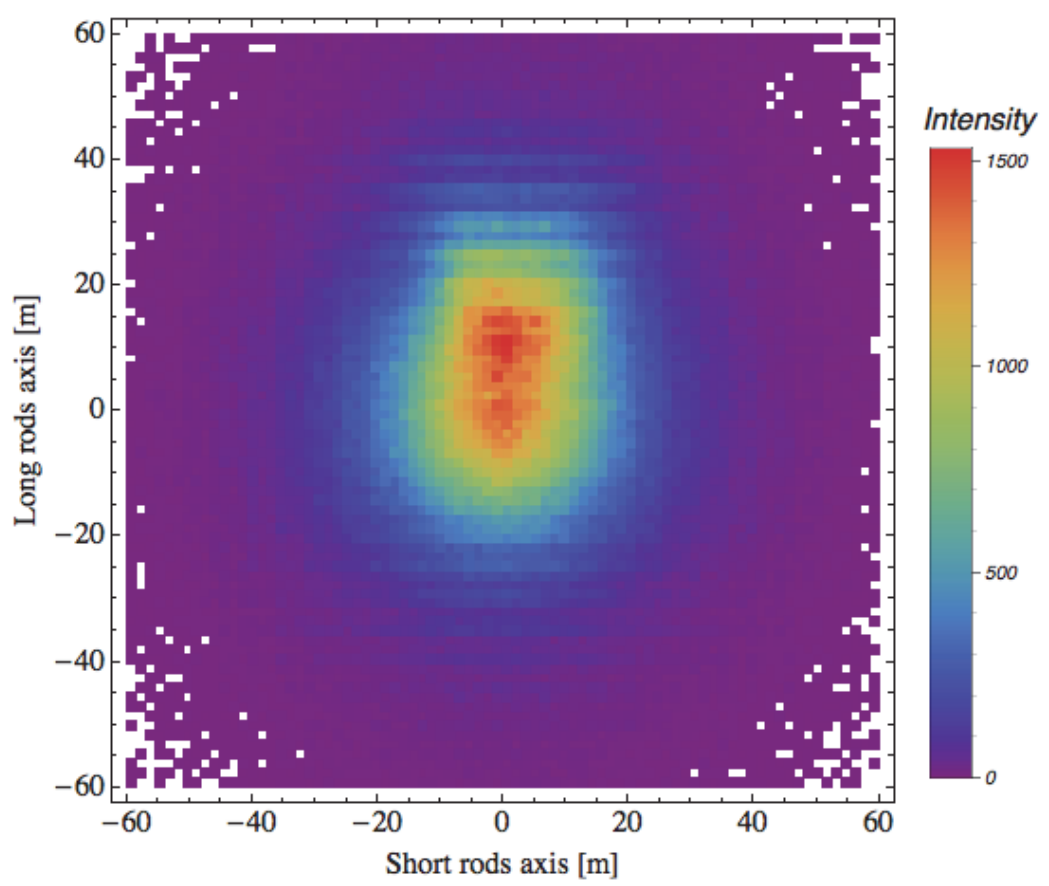

Figure 15. Density histogram of the muon angular vectors projected onto a plane 30 meters above the detector.

It is clear from Figure 15 that the muon flux intensity is highest for vertical incidence. If the muon spectrum were uniform in intensity, and the BMD were above ground, this plot would be homogeneous, reflecting only the detector acceptance angle in the processed data, which would show no muon intensity at angles past the maximum acceptance. Because the cosmic muon spectrum strongly favors more vertical muon flux, all projection plots will exhibit the intensity pattern observed here.

Clearly visible in this plot is the striated region above and below the center of the image. This arises due to the intrinsic resolution limitations of the BMD because of the bar size. Larger bars would give rise to more prominent striations. As previously discussed, when only a single bar in a layer is struck by a muon, a randomized bar position is assigned, which decreases the plotted striations significantly.

\section{Data collected in the PNNL Shallow Underground Laboratory}

The BMD was assembled and made operational at the University of Hawaii. It was subsequently sent to PNNL for testing above ground and in the PNNL Shallow Underground Laboratory. The data rate at the surface at PNNL was 5.2 events per second compared to 3.8 events per second in Hawaii. Both locations are about $100 \mathrm{~m}$ above sea level.

The placement of the BMD in the PNNL Shallow Underground Laboratory at a depth of approximately 35 meters-water-equivalent (mwe) was next to a stairway to allow for variation in the expected muon flux depending on angle. Data were obtained constituting 57 data runs with 560000 events. The average event rate was 1.0 event per second. The angular distributions and the distributions of hits across the bars appeared consistent with the above ground data. The total angular distribution was somewhat broader for the underground data due to the presence of the stairway near one end skewing the distribution. Figure 16 shows a 3-dimensional plot of angle in the $\mathrm{x}$ and $\mathrm{y}$ plane measured by the BMD above ground and below 
ground. The data has been smoothed to reduce the effect from the finite size of the bars used to measure the angle. The below ground data show an enhancement in rate toward one end compared to the distribution above ground due to the presence of the stairway. Above ground data had a mean bin uncertainty of $5.6 \%$ for bin intensities above 0.0005 , and $10.7 \%$ for bin intensities below. Underground data had a mean uncertainty of $3.5 \%$ for bin intensities above 0.0005 , and $8.1 \%$ for bin intensities below that.

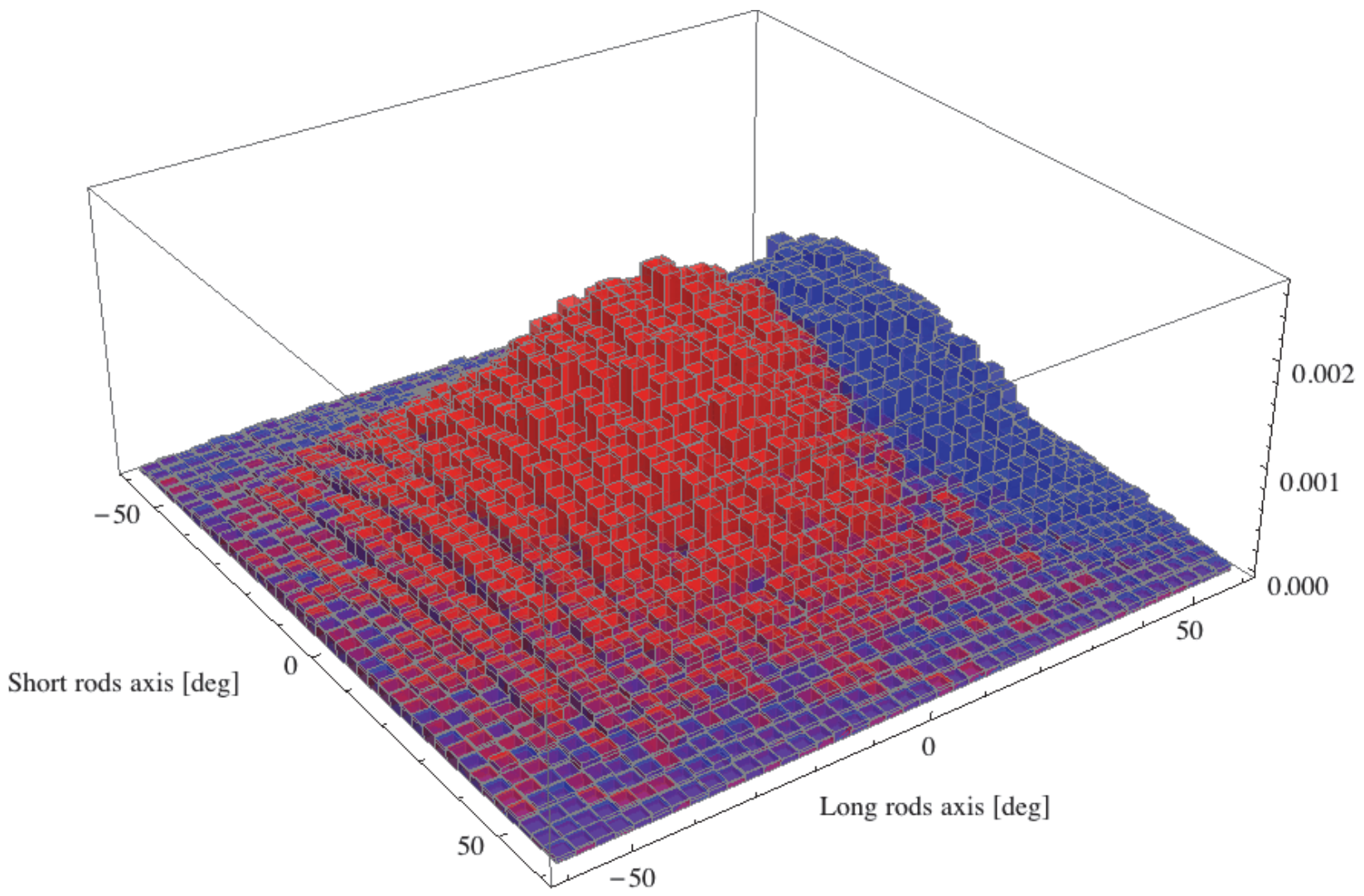

Figure 16. Histograms of $x$ and $y$ angles above ground (red) and below ground (blue). The vertical axis is intensity in arbitrary units.

Another direct comparison can be made between the above ground data and below ground data using a projection plot to a plane $30 \mathrm{~m}$ above the BMD surface (Figure 17). 

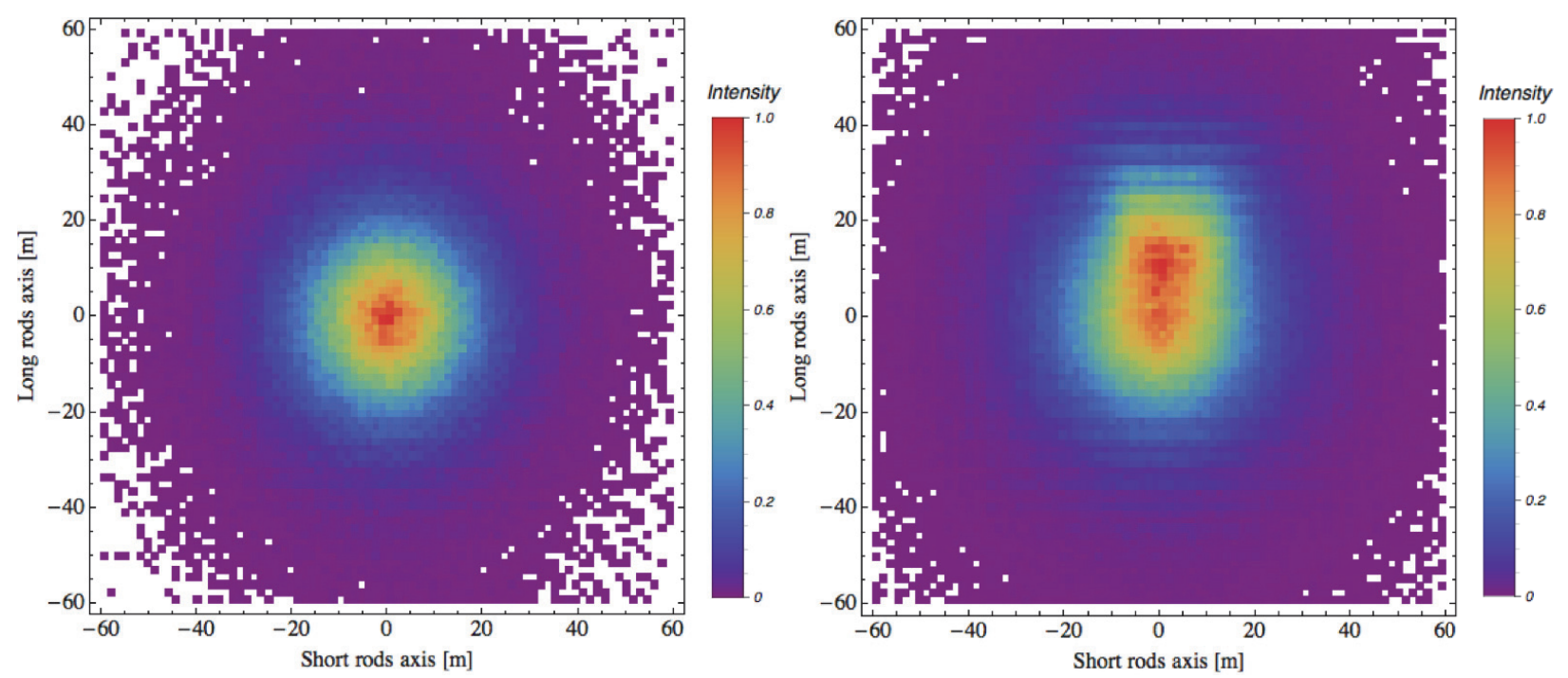

Figure 17. Left: Projection plot of the PNNL above ground data. A clear symmetry in muon flux can be seen. Right: Projection plot of the PNNL Shallow Underground Laboratory data with the asymmetry at the top, pointing toward the stairwell.

The asymmetry seen in the underground data (shown on the right in Figure 17) arises from actual asymmetries in the overburden of the lab. On one side there is a significant overburden, which remains fairly constant, but on the other there is a stairwell that allows for a significantly increased muon flux.

The simulations of the PNNL facility indicated there would be an asymmetry from the stairwell close to the BMD location. Figure 18 shows the angular muon flux collected by the prototype (left) and the simulation (right). The simulation exhibits good correlation with the data, with a flux hotspot centered at approximately $-0.5 \mathrm{rad}$ in the long rod axis $\left(\theta_{\mathrm{y}}\right)$.
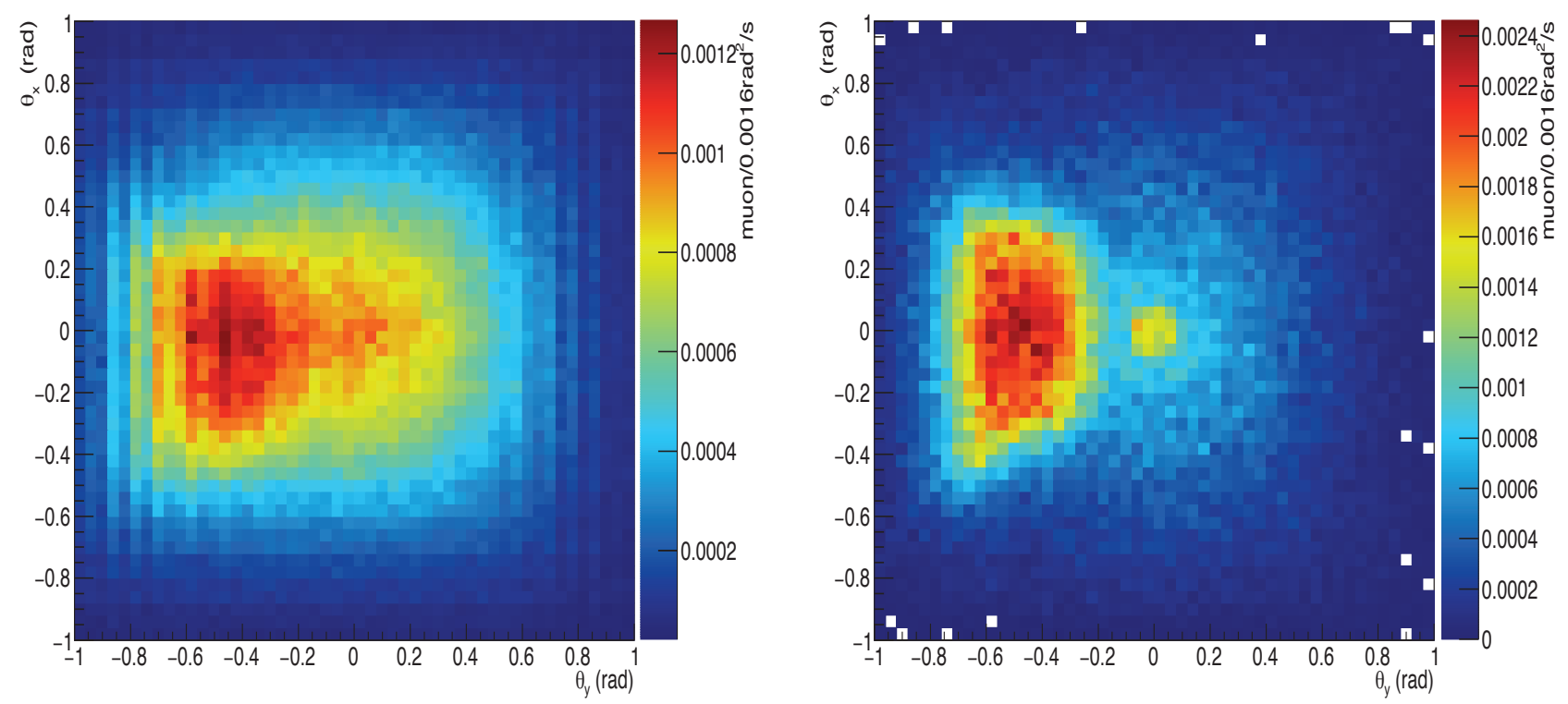

Figure 18. Angular muon flux obtained at the bottom of the PNNL Shallow Underground Laboratory from the prototype BMD (left) and from the simulation (right). The stairwell is to the left in these plots. 


\section{Results at LANL}

The prototype BMD was taken to LANL (approximately $2230 \mathrm{~m}$ above sea level) to be tested in the TA41 tunnel (Figure 10). Measurements were made in a laboratory building and then the detector was placed in the tunnel at five locations, as listed in Table 1. The tunnel has an outer garage door and an inner entry doorway enclosing a vestibule. Distance measurements in the tunnel were made relative to the entry door. The table lists each BMD location, approximate depth below the surface at that point, rate of muon events estimated from the Monte Carlo model and the measured rate, approximate measurement time, and total number of events obtained. At each location, the detector was rotated for equal durations with the "positive" end of the detector toward the end of the tunnel and the "negative" end toward the end of the tunnel.

Table 1. Results from measurements with the BMD at LANL.

\begin{tabular}{|c|c|c|c|c|c|c|}
\hline $\begin{array}{c}\text { Distance from } \\
\text { Door } \\
(\mathbf{m})\end{array}$ & $\begin{array}{c}\text { Position \# } \\
\text { in tunnel }\end{array}$ & $\begin{array}{c}\text { Approximate } \\
\text { Depth } \\
\mathbf{( m )}\end{array}$ & $\begin{array}{c}\text { Model } \\
\text { Estimated } \\
\text { Count Rate } \\
\mathbf{( H z )}\end{array}$ & $\begin{array}{c}\text { Measured } \\
\text { Count Rate } \\
\mathbf{( H z})\end{array}$ & $\begin{array}{c}\text { Measurement } \\
\text { Time } \\
\text { (days) }\end{array}$ & $\begin{array}{c}\text { Total } \\
\text { Events } \\
\text { Obtained }\end{array}$ \\
\hline Lab Room C101 & NA & 0 & 10 & 9.8 & 1 & $128 \mathrm{k}$ \\
\hline-1.7 & Outside & 0 & 9.0 & 8.0 & 1 & $87 \mathrm{k}$ \\
\hline 10 & 3 & 14 & 2.8 & 3.2 & 1.5 & $179 \mathrm{k}$ \\
\hline 35 & 2 & 40 & 0.8 & 0.88 & 4 & $339 \mathrm{k}$ \\
\hline 62 & 0 & 59 & 0.2 & 0.30 & 16 & $401 \mathrm{k}$ \\
\hline 78 & 1 & 74 & 0.14 & 0.19 & 20 & $240 \mathrm{k}$ \\
\hline
\end{tabular}

The prototype BMD data obtained was compared to the simulation data in Figure 19, which shows the muon flux predicted by the simulations and the actual flux obtained with the prototype BMD. The total expected flux from the simulation was 0.65 muons/s, while the total measured flux was 0.83 muons/s. 

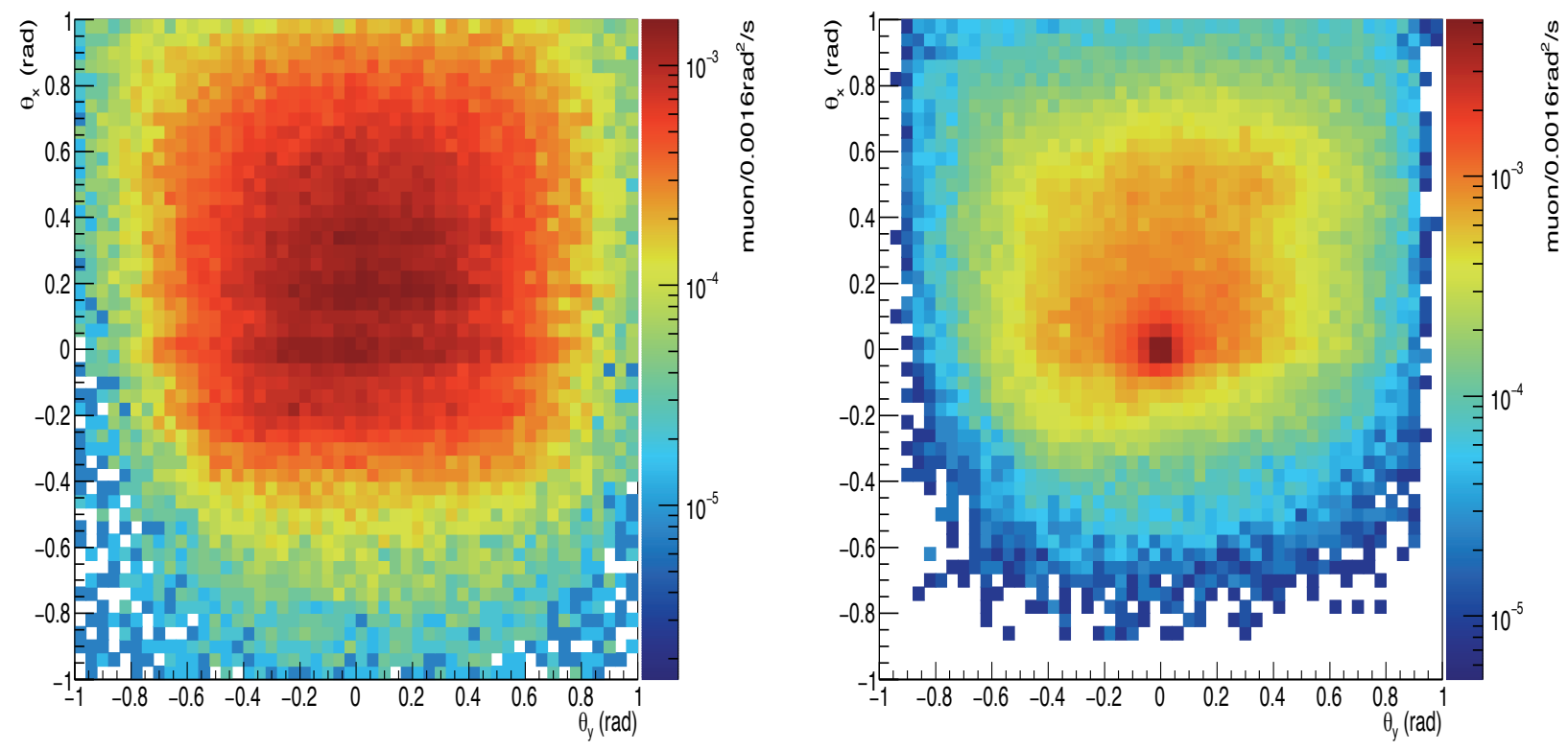

Figure 19. Angular muon flux for the prototype BMD (left) and the simulation (right). The tunnel entrance is toward the top.

In addition to the BMD, data were also acquired at the LANL Tunnel with a large muon detector built at LANL. The Mini Muon Tracker (MMT) has 12 layers of drift tubes covering an area of about $1.2 \mathrm{~m}$ square [14][15]. Data were acquired with the MMT at the same locations used subsequently for the BMD.

Flux plots were produced on similar axes to provide a direct comparison between the BMD and MMT detectors. The primary differences between the two detectors are the large size and 100-degree acceptance angle of the MMT, as compared to the BMD, which has a high acceptance angle of 130 degrees in the minor axis, but a surface area only approximately 32 times smaller than the MMT providing significantly lower count rates. Figure 20 presents flux plots (in normalized units of muons per unit area per unit time), hence they account for exposure time and histogram bin area when computing the flux. The ratio between the MMT and BMD count rates ranged from 11:1 to 14:1 depending on location. These values represent a cumulative effect of the detector areas (ratio of $\sim 32$ ), angular acceptance, and other confounding variables that might affect the threshold. Figure 20 shows plots where the rates from the MMT were normalized by a factor of 15 to be directly comparable to the BMD plots. Location 1 was farthest into the tunnel, which was the deepest underground location. The $0,2,3$ locations are progressively more shallow locations (Table 1). Each plot shows a projection of the muon tracks obtained onto a plane $100 \mathrm{~m}$ above the detector surface (roughly the top of the cliff). Each data set is then normalized for exposure time and area. The results show obvious parallels between the detectors, but the plots produced using the MMT detector data have higher resolution due to at least an order of magnitude more counts at each location. 
MMT
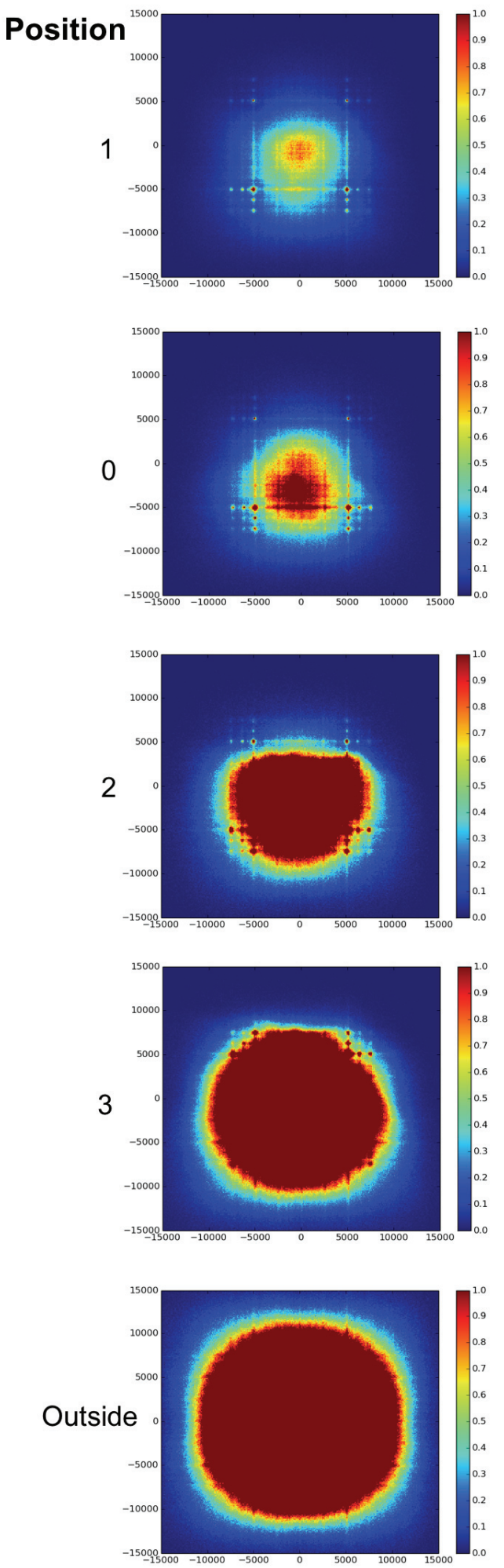

BMD
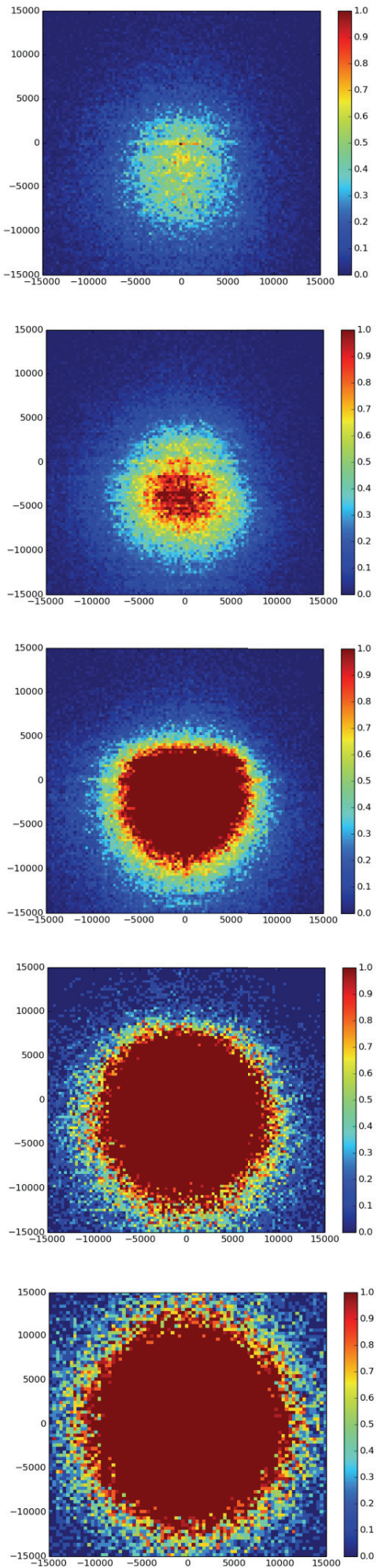

Figure 20. Comparison of the data from five locations measured by the MMT and BMD at LANL. Positions 1, 0, 2, and 3 are locations inside of the TA-41 tunnel at decreasing depth, with Position 1 being deepest (see Table 1). 


\section{Conclusions}

The BMD, a muon-tracking detector designed for use in boreholes for applications such as monitoring $\mathrm{CO}_{2}$ sequestration sites, was developed and tested. The BMD was successful in detecting muons above ground and in Shallow Underground Laboratory at PNNL and LANL. Results from the BMD compare well with those from the larger MMT detector, and demonstrate the feasibility of making angular measurements of overburden density at the precision required for subsurface applications. The next step will be to develop a complete instrument packaged into a stainless steel housing for actual testing in a borehole environment. While the prototype BMD demonstrates the concept for a borehole detector, it is somewhat too large to fit in a $17.8-\mathrm{cm}$ (7') borehole. The BMD will also require that the orientation be controlled carefully to optimize the cross sectional area to the downward going muons observed in a horizontal borehole underground. A new design concept has been developed for this next generation muon detector. It will fit into a pressure vessel with an outside diameter of $15.25 \mathrm{~cm}(6 ")$ and thus could be deployed in wells cased with $17.8 \mathrm{~cm}$ (7”) casings.

\section{Acknowledgements}

This work has been funded by the U.S. Department of Energy SubTER cross-cut program as a FY15FY16 sapling project. Battelle operates Pacific Northwest National Laboratory for the United States Department of Energy under contract DE-AC05-76RLO 1830. We thank Anna Pla-Dalmau of Fermi National Laboratory for providing the polystyrene rods used in this work. This is PNNL technical report PNNL-SA-120937 and Los Alamos publication number LA-UR-16-27062.

\section{References}

[1] T. Aguayo, J.E. Fast, R.T. Kouzes, J.L. Orrell, The mu-Witness Detector: A ruggedized, portable, flux meter for cosmogenic activation monitoring, IEEE Transactions on Nuclear Science, 60 (2013) 689-692.

[2] V.A. Kudryavtsev, N.J.C. Spooner, J. Gluyas, C. Fung, M. Coleman, Monitoring subsurface $\mathrm{CO}_{2}$ emplacement and security of storage using muon tomography, International Journal of Greenhouse Gas Control, 11 (2012) 21-24.

[3] H.K.M. Tanaka, T. Nakano, S. Takahashi, J. Yoshida, M. Takeo, J. Oikawa, T. Ohminato, Y. Aoki, E. Koyama, H. Tsuji, K. Niwa, High resolution imaging in the inhomogeneous crust with cosmic-ray muon radiography: The density structure below the volcanic crater floor of Mt. Asama, Japan, Earth Planet. Sci. Lett, 263, (2007) 104-113.

[4] H. Tanaka, K. Nagamine, N. Kawamura, S.N. Nakamura, K. Ishida, K. Shimomura, Development of the cosmic-ray muon detection system for probing internal-structure of a volcano, Hyperfine Interactions, 138 (2001) 521-526.

[5] N. Lesparre, D. Gibert, J. Marteau, Y. Déclais, D. Carbone, E. Galichet, Geophysical muon imaging: feasibility and limits, Geophysical Journal International, 183 (2010) 1348-1361.

[6] C.L. Morris, C.C. Alexander, J.D. Bacon, K.N. Borozdin, D.J. Clark, R. Chartrand, C.J. Espinoza, A.M. Fraser, M.C. Galassi, J.A. Green, J.S. Gonzales, J.J. Gomez, N.W. Hengartner, G.E. Hogan, A.V. Klimenko, M.F. Makela, P. McGaughey, J.J. Medina, F.E. Pazuchanics, W.C. Priedhorsky, J.C. Ramsey, A. Saunders, R.C. Schirato, L.J. Schultz, M.J. Sossong and G.S. Blanpied, Tomographic imaging with cosmic ray muons, Science \& Global Security, 16 (2008) $37-53$. 
[7] J D. Bacon, D.L. Chichester, J.M. Durham, J. Fabritius, S. Fellows, E. Guardincerri, D. Morley, C.L. Morris, K. Plaud-Ramos, D. Poulson, P. Winston, Cosmic ray muon imaging of spent nuclear fuel in dry storage casks, J. Nuclear Material Management, 44 (2016) 5-12.

[8] D.M. Mei, A. Hime, Muon-induced background study for underground laboratories, Phys. Rev. D, 73 (2006) 1-18.

[9] F.E. Gray, C. Ruybal, J. Totushek, D.M. Mei, K. Thomas, C. Zhang, Cosmic ray muon flux at the Sanford Underground Laboratory at Homestake, Nucl. Instruments Methods Phys. Res. A, 638 (2011) 63-66.

[10] S. Agostinelli, J. Allison, K. Amako, J. Apostolakis, H. Araujo, P. Arce, M. Asai, D. Axen, S. Banerjee, G. Rodrand, F. Behner, L. Bellagamba, J. Boudreau, L. Broglia, A. Brunengo, H. Burkhardt, S. Chauvie, J. Chuma, R. Chytracek, G. Cooperman, G. Cosmo, P. Degtyarenko, A. Dell'Acqua, G. Depaola, D. Dietrich, R. Enami, A. Feliciello, C. Ferguson, H. Fesefeldt, G. Folger, F. Foppiano, A. Forti, S. Garelli, S. Giani, R. Giannitrapani, D. Gibin, J. J. Gomez Cadenas, I. Gonzalez, G. Gracia Abril, L. G. Greeniaus, W. Greiner, V. Grichine, A. Grossheim, P. Gumplinger, R. Hamatsu, K. Hashimoto, H. Hasui, A. Heikkinen, A. Howard, V. Ivanchenko, A. Johnson, F. W. Jones, J. Kallenbach, N. Kanaya, M. Kawabata, Y. Kawabata, M. Kawaguti, S. Kelner, P. Kent, T. Kodama, R. Kokoulin, M. Kossov, H. Kurashige, E. Lamanna, T. Lampen, V. Lara, V. Lefebure, F. Lei, M. Liendl, W. Lockman, F. Longo, S. Magni, M. Maire, E. Medernach, K. Minamimoto, P. Mora de Freitas, Y. Morita, K. Murakami, M. Nagamatu, R. Nartallo, P. Nieminen, T. Nishimura, K. Ohtsubo, M. Okamura, S. O'Neale, Y. Oohata, K. Paech, J. Perl, A. Pfeiffer, M.G. Pia, F. Ranjard, A. Rybin, S. Sadilov, E. Di Salvo, G. Santin, T. Sasaki, N. Savvas, Y. Sawada, S. Scherer, S. Sei, V. Sirotenko, D. Smith, N. Starkov, H. Stoecker, J. Sulkimo, M. Takahata, S. Tanaka, E. Tcherniaev, E. Safai Tehrani, M. Tropeano, P. Truscott, H. Uno, L. Urban, P. Urban, M. Verderi, A. Walkden, W. Wander, H. Weber, J.P. Wellisch, T. Wenaus, D. C. Williams, D. Wright, T. Yamada, H. Yoshida and D. Zschiesche, Geant4-a simulation toolkit, Nucl. Instruments Methods Phys. Res. A, 506 (2003) 250-303.

[11] J. Flygare, A. Bonneville, R. Kouzes, J. Yamaoka, A. Lintereur, Muon Borehole Detector for Use in 4D Tomographic Density Monitoring, Submitted to IEEE Transactions on Nuclear Science, 2016 .

[12] C. Hagmann, D. Lange, D. Wright, Cosmic-ray shower generator (CRY) for Monte Carlo transport codes, IEEE Nucl. Sci. Symp. Conf. Record, Honolulu, HI (2007) 1143-1146.

[13] McGehee, E.D., K.L.M. Garcia, Historical Context of W Site, Technical Area 41, Los Alamos National Laboratory LA-UR-04-6492 (2004).

[14] L. Cuellar, K. Borozdin, K. Chung, A.J. Green, N. Hengartner, C. Morris, L.J. Schultz, N.P. Reimus, J.D. Bacon, W. Vogan-McNeil, A range muon tomography performance study for detection of explosives, Los Alamos National Laboratory LA-UR-1003500 (2010).

[15] J.O. Perry, Advanced applications of cosmic-ray muon radiography.” Dissertation at The University of New Mexico (2013). 